\title{
Nutritional Amblyopia Combined with Night Blindness
}

\author{
Angeline M. Nguyen Ashley A. Campbell \\ Richard D. Semba \\ Wilmer Eye Institute, Johns Hopkins University School of Medicine, Baltimore, \\ Md., USA
}

\section{Key Words}

B complex vitamins - Nutritional amblyopia $\cdot$ Night blindness $\cdot$ Vitamin A

\begin{abstract}
We report the case of an 18-year-old male who developed both nutritional amblyopia and night blindness. After nearly a lifetime of consuming a bizarre diet limited to French fries, pretzels, crackers, and carbonated sodas, he had a relatively sudden onset of night blindness and bilateral visual loss. The night blindness resolved after taking daily oral vitamin A supplements. Visual acuity gradually improved from light perception, both eyes, to 20/20 right eye and 20/25 left eye after multivitamin supplementation and vitamin $B_{12}$ injections. The patient had bilateral optic atrophy and bilateral ring scotomas around a small area of fixation. The patient was unable to modify his diet despite professional advice and counseling.
\end{abstract}

\section{Introduction}

Nutritional amblyopia is a retrobulbar optic neuropathy with bilateral visual loss, central or cecocentral scotomas, and dyschromatopsia generally attributed to the deficiency of B complex vitamins [1], although deficiencies in other nutrients may possibly play a role. The disease has mostly been reported among alcoholics, prisoners of war, inmates in jails, and in populations following natural disasters. We report a case of nutritional amblyopia combined with night blindness from vitamin A deficiency in a young adult on a bizarre diet. To our knowledge, this is the first report of combined nutritional amblyopia and night blindness.

\section{Case Report}

An 18-year-old male presented at an outside hospital with a chief complaint of sudden onset of poor vision and difficulty driving at night in mid-May 2005. He participated regularly in baseball as an umpire. During an evening game, he noticed that he could not see a line drive down first base, 
although others around him could see the ball. After the game, he drove home, but once he was beyond the lights of the stadium, he could not see anything and stopped his car. He called a friend and was given a ride home. Within several days, his vision loss reportedly progressed to light perception, OU, as reported by his general ophthalmologist in Pittsburgh. No visual fields were obtained. The general ophthalmologist did not note any other neurological signs or symptoms. In mid-June 2005 , the patient was seen by a neuro-ophthalmologist, who noted bilateral optic atrophy and otherwise normal retinas. Serum retinol concentrations were undetectable $(<2 \mu \mathrm{g} / \mathrm{dl})$, serum $\mathrm{B}_{12}$ was 181 $\mathrm{pg} / \mathrm{ml}$, serum folate was $13.3 \mathrm{ng} / \mathrm{ml}$, and complete blood count was normal. Magnetic resonance imaging studies of the orbit and brain were normal. Goldmann visual fields showed subtotal visual field loss, OU. No neurological signs or symptoms were mentioned in the previous records. The patient was placed on vitamin A, 50,000 IU/day, multivitamins, and vitamin $\mathrm{B}_{12}$ injections, and his night blindness resolved. By mid-July 2005, his vision improved to 20/30 OD and CF $2 \mathrm{ft}$ OS.

In August 2005, he presented for the first time at the Wilmer Eye Institute, where he was examined by one of the co-authors (R.D.S.). His visual acuity was 20/60 OD and CF $2 \mathrm{ft}$ OS. Ishihara plates were $3 / 14$ OS and 0/14 OS. The patient had temporal disc pallor OU, more pronounced in the left than the right eye (fig. 1). Goldmann visual fields showed central scotomas, OU. The patient had a body mass index of $30.5 \mathrm{~kg} / \mathrm{m}^{2}$. The patient and his mother reported that he has had a diet for the last 14-16 years that consisted only of French fries, pretzels, crackers, and carbonated sodas. He did not eat any fruit or vegetables, meat, dairy products, grains, or nuts. In childhood, the patient had been diagnosed with obsessive-compulsive disorder, a condition that may have contributed to his refusal to diversify his unbalanced diet despite the concern of his family and advice of his doctors. The patient was advised at the time of his first visit to the Wilmer Eye Institute to start eating more fruit, vegetables, dairy products, legumes, fish, beef, and poultry as per US dietary guidelines.

From August 2005 until March 2009, his visual acuity improved to 20/20 OD and 20/25 OS. Ishihara plates remained $3 / 14$ OS and 0/14 OS. Humphrey visual field examination in 2005 revealed a small central scotoma OD and a large central scotoma OS (fig. 2). Amsler testing showed a small area of central fixation surrounded by a ring scotoma in each eye. The outside diameters of the scotomas slowly increased in size from 2005 to 2009 and then stabilized. During this period, the patient reported fluctuations in his vision over the course of a day, with his vision being better at the beginning of the day. The patient was unable to read as quickly as he had done prior to 2005 . He also reported difficulty seeing anything written in pencil. In 2008, the patient developed numbness and tingling in his extremities and was diagnosed by his neurologist with subacute combined degeneration of the spinal cord. The patient was repeatedly encouraged to seek counseling to help him with the eating disorder. He was unable to make any substantial changes to his diet.

He continued to take vitamin A, 10,000 IU per day, monthly vitamin $\mathrm{B}_{12}$ injections, and two multivitamins per day. In October 2009, a pattern visual evoked potential showed marked abnormalities in cone-mediated central retinal processing and/or the pathway from the retina to the visual cortex in both eyes. A full-field electroretinogram (ERG) showed normal a waves in both eyes, and $b$ waves that were delayed in both eyes in dark-adapted conditions. The b/a wave amplitude ratio was normal in both eyes. The oscillatory potentials were severely abnormal in both eyes. The full-field ERG was consistent with a moderate bilateral retinopathy in which cone-mediated responses were compromised but rod-mediated responses were normal. Despite his ocular problems, the patient finished an undergraduate degree at a well-known national university and received a graduate degree from a prestigious graduate school.

\section{Discussion}

Both vitamin A deficiency and nutritional amblyopia are rare in industrialized countries. Vitamin A is an essential precursor for rhodopsin in the visual cycle, and lack of vitamin A leads to night blindness. Vitamin $B_{12}$ is essential for normal hematopoiesis and neurological function, and lack of vitamin $\mathrm{B}_{12}$ can result in megaloblastic anemia and subacute combined degeneration. When nutritional amblyopia occurs in industrialized countries, it has been primarily described in alcoholics [1]. The usual treatment for nutritional amblyopia consists of adopting a proper diet that includes 
foods rich in thiamin (e.g. whole-grain breads), niacin (e.g. meat, fish, poultry), folate (e.g. beans, green leafy vegetables), and vitamin $B_{12}$ (e.g. meat, poultry, whole milk). In this case, dietary modification was extremely difficult. This condition is typically reversible if treated with a proper diet and adequate vitamin supplementation within 2 or 3 months of onset of visual loss, but in this case the patient sustained permanent major visual field loss. The ring scotomas were an unusual aspect of this case.

\section{Disclosure Statement}

Supported in part by a Lew Wasserman Merit Award to Dr. Semba from Research to Prevent Blindness.

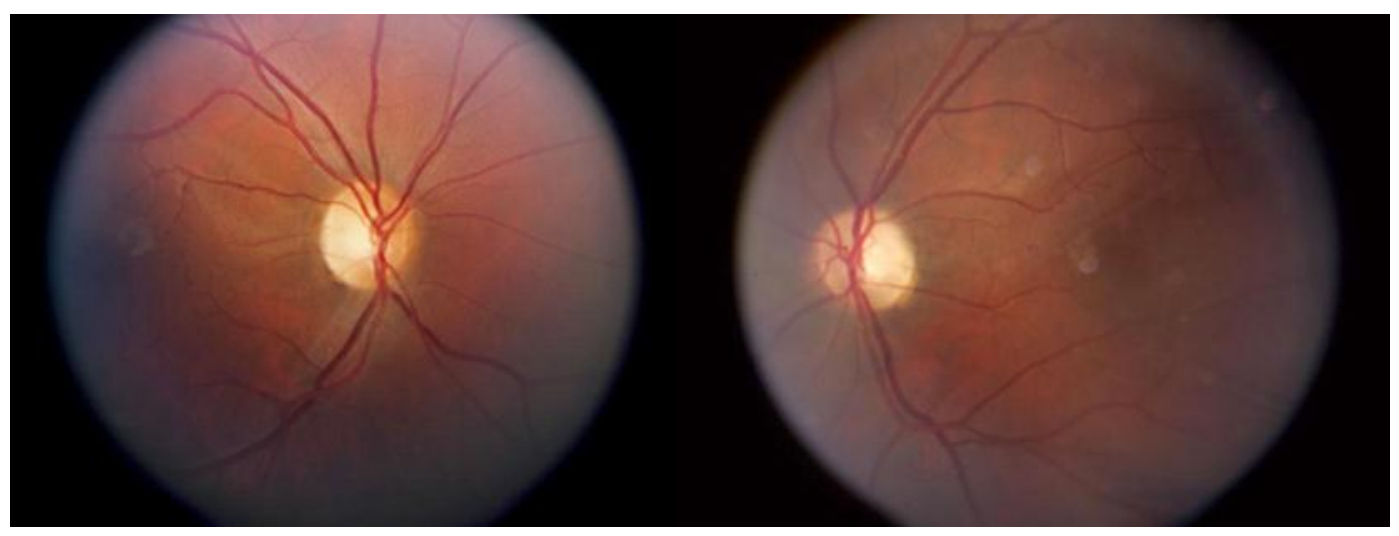

Fig. 1. Fundus photographs showing temporal disc pallor, OU.
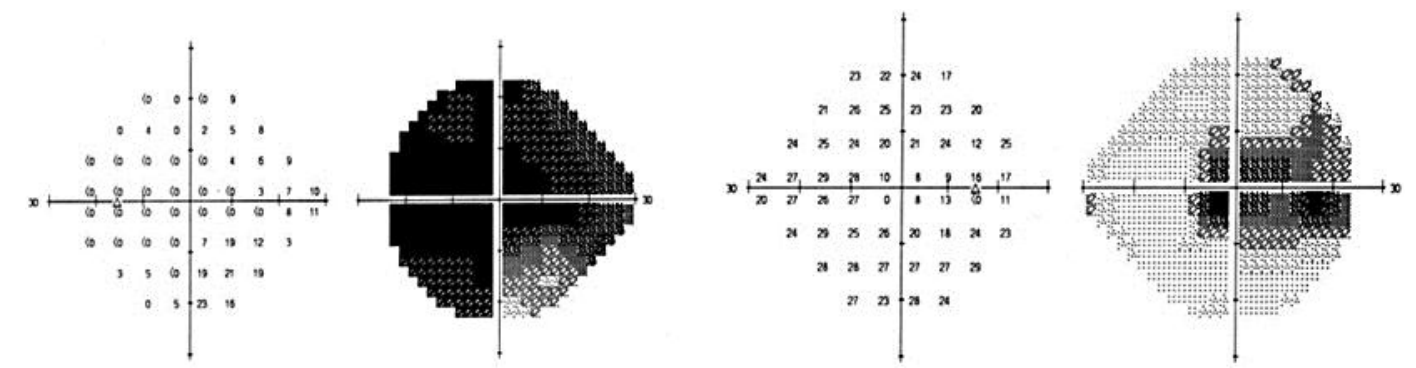

Fig. 2. Humphrey visual field, 24-2, showing a small central scotoma OD and a large central scotoma OS.

\section{Reference}

1 Semba RD: Handbook of Nutrition and Ophthalmology. Totowa, Humana Press, 2007, pp 281-354. 